\title{
Molecular techniques for clinical diagnostic virology
}

Steven J Read, David Burnett, Colin G Fink

A decade after the first studies were performed it is justifiable to claim that molecular techniques have revolutionised the work of the clinical virology laboratory. Hitherto, the role of the virology laboratory was often a retrospective diagnosis based on virus isolation and serology. Nevertheless, the epidemiological data collected in this way justified the continued activity of clinical virology. The recent molecular revolution in laboratory methods has been timely because it has been in parallel with the emergence of new pathogens that have presented the clinical virologist with fresh diagnostic challenges. A concurrent development of specific antiviral compounds has increased the potential of rapid laboratory investigation to contribute to the management of acutely ill or immunosuppressed patients.

Molecular assays for the detection of microorganisms can be designed even when only partial nucleic acid sequence information is available. This is valuable when identifying and diagnosing new diseases and emerging pathogens because there is the possibility of a rapid development of assays in house. However, the standardisation of assay design is a continuing problem because of the large number of nucleic acid sequences that can be used as a target for the detection of any one virus. Many of the methodologies associated with molecular techniques have now been incorporated into commercial kits, but in house molecular assays continue to be used. In house assays are often well evaluated and perform to a high standard, but differing assay sensitivities and specificities have been highlighted by quality assessment programmes. Some of the variations in performance can be accounted for by assay design, although it is probable that some in house assays do not travel well and perform badly because laboratory staff are inexperienced with molecular techniques. Molecular techniques in their present format, even in commercial kits, require a considerable degree of operator skill. If a wider use of molecular techniques in a district hospital setting is considered desirable, more robust protocols that consider the extreme sensitivity of amplification assays, together with more standardisation of methodology, will be required. Most in house assays are unsuited to high throughput testing and are demanding of staff time; these problems are now being dealt with by developments in assay automation.

\section{Methodologies}

TARGET AMPLIFICATION TECHNIQUES

Dr Read

email:

micropathology@telinco.co.uk

Accepted for publication 10 November 1999 an enzymatic step to amplify the target nucleic acid before detection of the specific sequence.
Clinical material may be rich in human genomic DNA, but it is possible to amplify only a very few target viral genomes present to become the dominant, easily detected sequence. A variety of target amplification techniques has been developed but those most widely used and commercially developed are the polymerase chain reaction (PCR), ligase chain reaction (LCR), and nucleic acid sequence based amplification (NASBA).

\section{PCR}

PCR was the first nucleic acid amplification technique to be described and, perhaps because of its widespread use in research applications, has become the most widely used molecular diagnostic technique in clinical virology. There are several reviews, chapters, and books devoted to its methodology and application. ${ }^{12}$ The exponential amplification of a single target sequence is theoretically possible so that exquisitely sensitive detection is achieved. PCR has been made more versatile by the development of several variants of the basic method; RNA genomes can be detected if first converted into a complementary DNA copy; several nucleic acid sequences can be detected simultaneously using a cocktail of primers; the technique can be made more sensitive and specific by using double amplification with appropriately designed "nested" primers, or the amplification may be made less specific to detect divergent genomes or partially characterised sequences.

\section{Alternative target amplification techniques}

The development of commercial assays has taken place only for relatively high throughput applications, such as the detection of Chlamydia trachomatis and human immunodeficiency virus (HIV). PCR is covered by patent rights and so the major commercial development of this technique has been restricted to one manufacturer. Neither of the alternative target amplification techniques is widely used as the basis for in house assays, but they have been developed for use in commercial kits because of the restrictions on PCR use. LCR, developed by Abbott Laboratories, uses a thermostable ligase to join two oligonucleotides to each other when they are hybridised to their respective target sequences. Amplification of nucleic acid sequences by LCR can be exponential because each round of synthesis can double the number of target molecules. NASBA, developed by Organon Teknika, has the advantage of being an isothermal process, so that thermal cycling equipment is not required. Compared with the other nucleic acid amplification methods, it is particularly suited to the detection of RNA 
viruses because an RNA polymerase is used to amplify RNA without conversion to complementary DNA.

\section{Signal amplification techniques}

These techniques do not replicate the nucleic acid detected in the assay but rather use amplification of a signal generated by detection of the target sequence. Branched DNA (bDNA) technology incorporated into commercial assays by Chiron Corporation uses multiple DNA probes, first for detection, and subsequently for increasing the number of potential binding sites from which the reporter signal is eventually generated. This approach is less sensitive than target amplification but has several advantages. The assay format is well suited to routine high throughput testing and it is not prone to false positives caused by cross contamination. It is therefore more readily suited for use in virology laboratories where staff are inexperienced in molecular techniques. Importantly, bDNA assays are capable of detecting a broader range of viral genotypes than PCR based assays, which with their more limited number of target sequences have a restricted specificity: potentially a problem when detecting viral RNA genomes, which can show high sequence heterogeneity. The bDNA based Quantiplex assays for the measurement of $\mathrm{HIV}$ and hepatitis $\mathrm{C}$ virus (HCV) viraemia measure more accurately a broader spectrum of viral genotypes than the first generation PCR based Amplicor assays. ${ }^{3}$

\section{Applications of molecular techniques} NEW APPROACHES TO THE DISCOVERY OF VIRUSES AND DISEASE AETIOLOGIES

Molecular techniques have been instrumental in the recent discoveries of viruses associated with hepatitis. ${ }^{4}$ The most important of these came in 1989 with the partial characterisation of the infectious agent associated with most cases of non-A non-B hepatitis. ${ }^{56}$ This condition usually presented as post-transfusion jaundice and was long suspected to have a viral aetiology. Nucleic acid from infectious chimpanzee blood was used to produce sufficient quantities of virus encoded proteins for the development of a diagnostic antibody assay. This molecular approach was successful in developing a laboratory diagnostic assay before the virus had been isolated and characterised in the conventional sense; now named hepatitis $\mathrm{C}$ virus, it has been characterised extensively using molecular techniques. Another virus has been similarly discovered and characterised, ${ }^{7}$ and although termed hepatitis $G$ virus, a role for this virus or group of viruses in human disease remains unclear. ${ }^{8}$

A powerful PCR based technique known as representational difference analysis has been used to discover a virus associated with cancer in HIV infected individuals. ${ }^{9}$ Apart from epidemiological data suggesting an infectious cause of Kaposi's sarcoma, there had been no characterisation of the newly discovered member of the herpesvirus group. Human herpesvirus 8 , or $\mathrm{KS}$ virus, has been detected in tissue from HIV infected individuals, including those with Kaposi's sarcoma lesions, ${ }^{10}{ }^{11}$ and also from sarcoid. ${ }^{12}$

\section{DIAGNOSTIC PROBLEMS}

Traditionally, laboratory diagnostic techniques in virology have relied on an ability to propagate infectious virus from clinical material in cell culture, or on the detection of a specific antibody. Where virus propagation is not successful, laboratory diagnosis has remained a problem because direct detection methods for viral antigen, nucleic acid, or morphology by microscopy in clinical material have low sensitivity. These difficulties, together with the time interval required to detect a specific immune response and the interpretation of antibody values for viruses that cause latent infections, has caused continuing diagnostic dilemmas for the clinical virologist. A reliable diagnosis of HIV and HCV infection is usually achieved by the detection of antibody; however, these viruses can be transmitted vertically and passive acquisition of maternal antibody confuses confirmation of infection in the neonate. The serological diagnosis of HIV and HCV infection is also impossible in the "window period" before seroconversion, and this poses a concern for the safety of blood products: an estimated risk of $\mathrm{HCV}$ transmission in western Germany is $1 / 20000$ for first time donors and $1 / 200000$ for repeat donors. ${ }^{13}$

Molecular assays in some circumstances might be no more sensitive or specific than traditional techniques, although they may be more suited to large scale screening programmes. The detection of $C$ trachomatis infection, an "atypical" organism that has traditionally been the interest of the virologist, has evolved from isolation in cell lines, which is technically difficult but has good specificity, to antigen detection by enzyme immunoassay or fluorescence antibody test, and now to molecular amplification of pathogen DNA. The commercial molecular assays that have been developed are more sensitive than antigen detection, ${ }^{14}$ and much better suited to routine use than cell culture isolation. The use of molecular detection has led to a growing awareness of the extent of chlamydial infection and the potential value of a comprehensive screening programme.

\section{CENTRAL NERVOUS SYSTEM INFECTION}

Central nervous system (CNS) viral infections, although recognised clinically, are a diagnostic difficulty because of the generally low sensitivity of virus isolation methods from cerebrospinal fluid (CSF). Herpes simplex virus type 1 (HSV-1) is rarely recoverable from the CSF of patients with herpes simplex virus (HSV) encephalitis, probably because relatively few viable virus particles are released into the CSF. For a while, techniques based on the detection of virus in brain biopsy were attempted to provide a reliable, prompt diagnosis in this illness, but the advent of effective antivirals of low toxicity has made invasive techniques difficult to justify. In cases of enterovirus meningitis, virus might be recovered from CSF, although not all 
serotypes grow in laboratory cell lines, and growth is generally too slow to aid differentiating between viral and bacterial meningitis. Hitherto, patients with viral CNS infection have often received only a presumptive diagnosis based on clinical presentation: without supportive laboratory evidence an understanding of the aetiology and epidemiology of viral CNS disease has been limited.

Molecular amplification techniques have been able to overcome many of these problems through the ability to detect just a few copies of viral nucleic acid directly in CSF. The use of the PCR technique for the diagnosis of HSV encephalitis was one of its first applications in the clinical virology laboratory, and when it was demonstrated that PCR could potentially provide sensitive and specific diagnosis, ${ }^{15}$ the test was evaluated extensively in several prospective studies. ${ }^{16-18}$ Assays for the other herpesviruses and enteroviruses have also been developed and found to contribute to laboratory diagnosis and patient management. ${ }^{19-22}$ The molecular amplification approach to the diagnosis of HSV encephalitis is now recognised as the method of choice for this disease, ${ }^{17}{ }^{23}$ and also for viral CNS infection in general. ${ }^{24}$ PCR analysis of CSF has demonstrated that symptoms of meningitis might be caused by the reactivation of

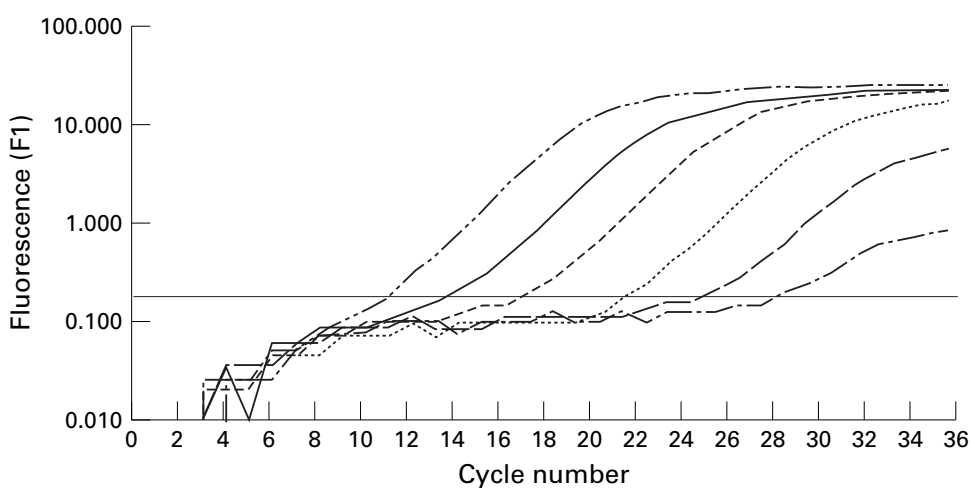

Figure 1 Measurement of hepatitis $B$ virus (HBV) genome in serum using real time monitoring of polymerase chain reaction (PCR) amplification kinetics of a set of standards on the LightCycler instrument. The PCR product is detected by a double stranded DNA specific fluorescent dye. Each line represents a PCR amplification. This experiment demonstrates the amplification of $H B V D N A$ from a $1 \log _{10}$ dilution series of the $H B V$ $D N A$ positive serum sample standard with an initial concentration of $10^{7}$ genomes $/ \mathrm{ml}$. The concentration of HBV genome is proportionate to the PCR cycle at which the fluorescent signal increases above the background level (represented by the horizontal line).

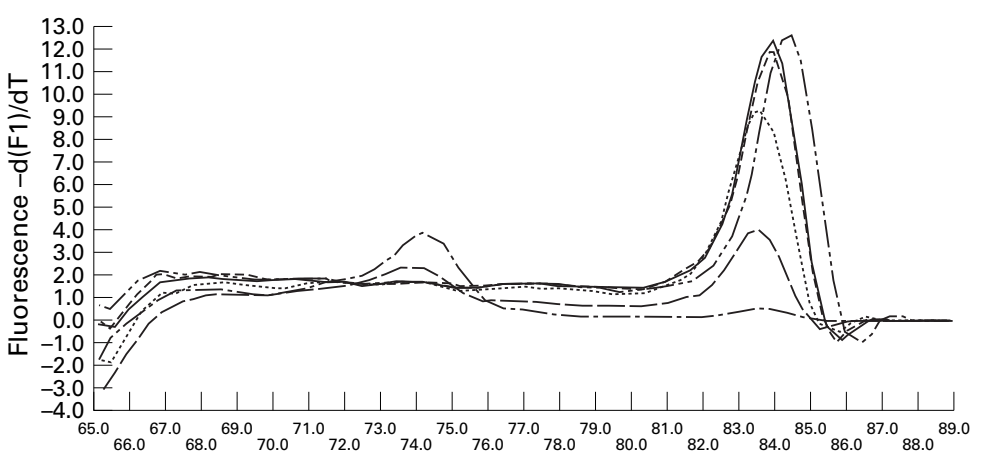

Temperature $\left({ }^{\circ} \mathrm{C}\right)$

Figure 2 Additional information about the reaction detailed in fig 1 can be obtained from the melting curve analysis. Analysis of the melting characteristics of the polymerase chain reaction (PCR) products demonstrates two distinct products: primer dimer, melting at $74^{\circ} \mathrm{C}$, and amplified hepatitis $B$ virus (HBV) product melting at $84^{\circ} \mathrm{C}$. Primer dimer formation occurs only at lowest input copy numbers. varicella zoster virus (VZV) ${ }^{25}{ }^{26}$ or herpes simplex virus type 2 (HSV-2) ${ }^{26}{ }^{27}$ infection without concurrent skin lesions. HSV-2 infection has been shown to be the cause of Mollaret's meningitis ${ }^{28}$ and an important cause of apparent sporadic aseptic meningitis; in one study, this virus was the most common aetiology found in women with this clinical diagnosis. ${ }^{29}$ Where traditional diagnostic methods are insensitive, our understanding of the natural history of viral disease continues to be improved through the use of molecular techniques, and in CNS infection our knowledge of the range of viral aetiologies has improved alongside the potential for early therapeutic intervention.

DIAGNOSIS IN IMMUNOSUPPRESSED PATIENTS

The development of antiviral drugs has highlighted the potential value to treatment of a rapid diagnosis of virus reactivation in immunosuppressed patients. Cytomegalovirus (CMV) is a major cause of post-transplant morbidity and, because it replicates only slowly in conventional cell culture, methods such as immunostaining for CMV early antigen expression have been used to speed the detection of virus replication in inoculated cell monolayers in the laboratory. Methods for detecting directly either virus specific antigen ${ }^{30}$ or nucleic acid ${ }^{31}$ in clinical material have removed the need for virus cell culture. An early concern that the high sensitivity of molecular amplification techniques would result in the detection of latent herpesvirus genome, so lowering the clinical specificity of virus nucleic acid detection, have been unfounded: the detection of viraemia has been found to correlate well with active CMV disease ${ }^{32} 33$ and CMV PCR is now often incorporated into routine post-transplant microbiological surveillance of susceptible patients.

\section{VIRAL GENOTYPING}

The problem of drug resistant virus phenotypes is becoming more recognised with an increase in the number of immunosuppressed patients receiving long term antiviral treatment. The use of prophylactic antiviral treatment for reactive herpesvirus infection, and the use of combinations of drugs to suppress viral replication in HIV infection, has selected for mutations conferring drug resistance. Under selective pressure, a drug resistant strain can quickly become the dominant phenotype, but molecular based assays have improved the clinical management of these patients by facilitating the detection of mutations conferring resistance. For the HIV genome, a commercial assay is available based on molecular amplification of the viral genome followed by hybridisation with mutation specific DNA probes. At present, these methods are used for the detection of resistance to the reverse transcriptase inhibitor class of antiviral compounds, but as a rapid and inexpensive approach it is likely to be used for other phenotypic determinants. A similar technique has been used for genotyping $\mathrm{HCV}$ infection for epidemiological study and for predicting the response to treatment. ${ }^{343}$ 
VIRAL LOAD

Molecular assays have had an important role in the study of the natural history of HIV infection, and subsequently in the development of effective treatment strategies. Quantitative molecular assays have shown that HIV-1 infection is characterised by high rates of virus production and clearance of both infected cells and cell free virions. ${ }^{36}$ This discovery led to the development of highly active antiretroviral treatment, the aim of which is the durable maximum suppression of virus replication. ${ }^{37}$ This advance in HIV treatment has been facilitated by the recent commercial development of viral load assays. The assessment of viraemia is now used as a prognostic indicator in $\mathrm{HIV}^{38}$ $\mathrm{HCV}^{39}$ and hepatitis $\mathrm{B}$ virus ${ }^{40}$ infections, and is also used in monitoring the efficacy of antiviral treatment in systemic viral infections of immunosuppressed patients. ${ }^{33}$

\section{Further developments}

The introduction of molecular techniques to routine diagnostic virology laboratories may have been held back by the lack of assays developed commercially, the high initial costs, and the specialist training involved in establishing these techniques. Using the technology currently available, the areas described might represent their limits in clinical virology; however, it is probable that formats currently under development and further genome sequencing will spread molecular detection more widely throughout diagnostic microbiology.

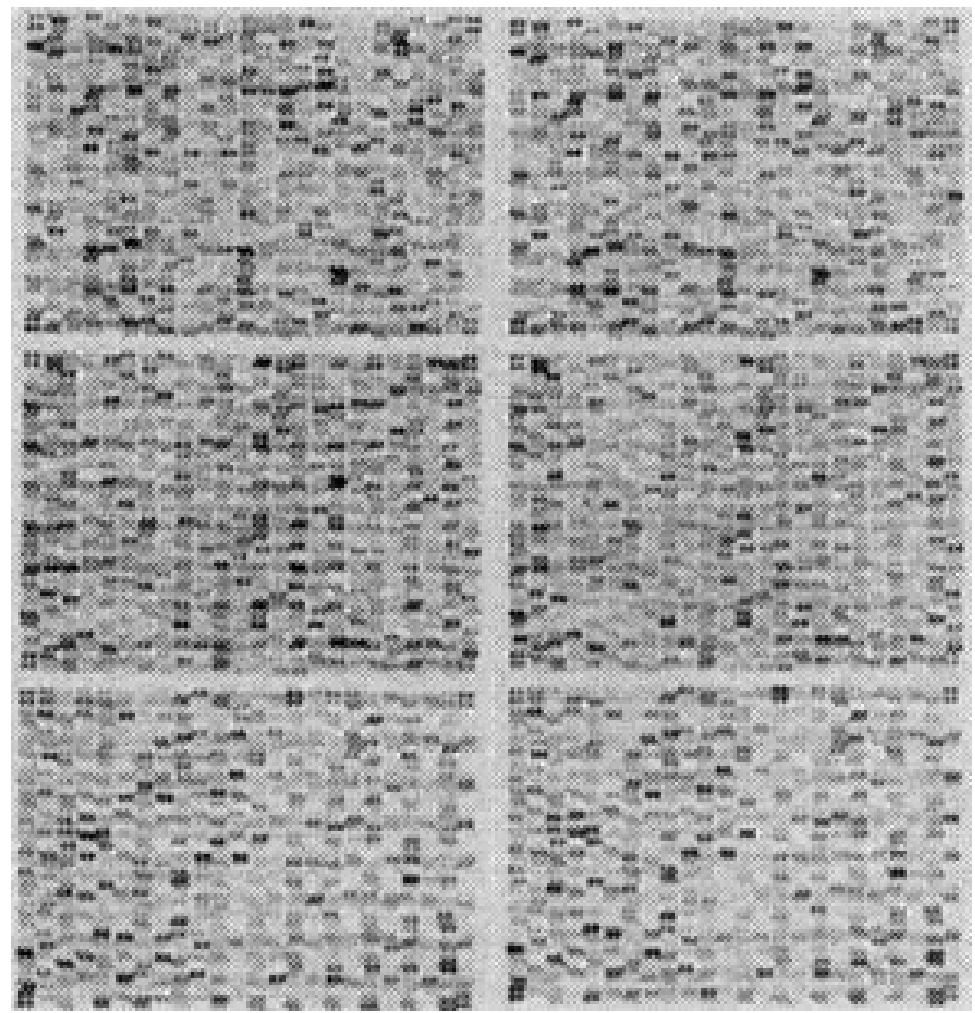

Figure 3 A "laboratory on a chip". A DNA chip containing immobilised arrays of oligonucleotides for the study of gene expression. Specialised equipment is required for interpreting the patterns generated by hybridisation of DNA to the array but the technique is suited to automated, high throughput applications.
AUTOMATED ASSAY FORMATS

Automation in virology laboratories for serology assays has been slow to transfer to molecular amplification techniques, but sequence detection and other downstream processes have now been automated successfully with the introduction of colorimetric based detection methods. However, if molecular assays are to become the methods of choice, there is a requirement for automated procedures for specimen preparation and nucleic acid extraction, which currently are "hands on" and time consuming. An important proposed development will be the ability to perform molecular assays on an automated platform currently widely used for serology assays. If these processes are automated successfully, it seems likely that for high throughput applications molecular assays will replace many traditional microbiological methods, which will become in contrast, both slow and expensive.

REAL TIME DETECTION AND CHARACTERISATION OF DNA SEQUENCES WITH FLUORESCENT DYES An exciting new development is the combination of thermal cycling for PCR nucleic acid amplification and fluorimetry in a single machine, such as the LightCycler instrument from Roche Molecular Biochemicals, able to support a number of fluorescent chemistries. It is possible by measurement of the signal from DNA sequence specific fluorescent dyes to monitor amplification kinetics in real time, allowing for accurate, rapid, and cheaper viral load tests (figs 1 and 2). These assays have the potential for accurate measurement over a wide range of viral concentrations. This instrument drastically reduces the problem of contamination in PCR because the reaction is analysed in a sealed system without exposure to the environment. Viral genotyping and mutation analysis can also be performed within the system by characterisation of the PCR product. The technology facilitates assay automation because the amplification, data collection, and analysis can be performed by a single machine connected to a computer.

SEQUENCE DETECTION AND CHARACTERISATION USING OLIGONUCLEOTIDE MICROARRAYS

Microarray "DNA chips" containing immobilised oligonucleotide probes can be used to screen rapidly for a number of target molecules amplified from clinical material (fig 3). Genetic screening has encouraged a rapid development of this technology, ${ }^{41}$ and microbiological application has recently been described in screening for drug resistance in tuberculosis. ${ }^{43}$ This technology might lead to the development of diagnostic kits for specific syndromes-for example, CNS infection, fever in immunosuppressed transplant recipients, genitourinary or upper respiratory tract infections. The use of this "syndromic" approach for the routine diagnostic laboratory would see a breakdown in the speciality barriers within microbiology laboratories because the techniques used for the detection of viruses, bacteria, fungi, and drug resistant genotypes all become based on the detection and characterisation of pathogen 
nucleic acid. This development, backed by advances in assay usefulness and reliability resulting from automation, might herald the end of the traditional stand alone clinical virology laboratory.

1 Baumforth KRN, Nelson PN, Digby JE, et al. The polymerase chain reaction. $\mathcal{F}$ Clin Pathol: Mol Pathol 1999;52:112ase

2 Becker Y, Darai G. PCR: protocols for diagnosis of human and animal virus diseases. Springer Lab Manual. Berlin: animal virus diseases.

3 Hawkins A, Davidson F, Simmonds P. Comparison of viral loads among individuals infected with hepatitis C virus
(HCV) genotypes 1,2 , and 3 by quantiplex HCV RNA assay versions 1 and 2 , Roche Monitor assay, and an in-house limiting dilution method. $\mathcal{F}$ Clin Microbiol in-house limitin

4 Gao SJ, Moore PS. Molecular approaches to the identification of unculturable infectious agents. Emerg Infect Dis 1997;2:159-67.

5 Choo QL, Kuo G, Weiner AJ, et al. Isolation of a cDNA clone derived from a blood-borne non-A, non-B viral hepatitis genome. Science 1989;244:359-62.

6 Alter HJ. Descartes before the horse: I clone, therefore I am: the hepatitis C virus in current perspective. Ann Intern Med 1991;115:644-9.

7 Simons JN, Leary TP, Dawson GJ, et al. Isolation of novel virus-like sequences associated with human hepatitis. Nat Med 1995;1:564-9.

8 Alter HJ, Nakatsuji Y, Melpolder J, et al. The incidence of tranfusion-associated hepatitis $G$ virus infection and its tranfusion-associated hepatitis $G$ virus infection and 1

9 Moore PS, Gao SJ, Dominguez G, et al. Primary characterisation of a herpesvirus agent associated with Kaposi's sarcoma 7 Virol 1996:70:549-58.

10 Strathdee SA, Veugelers PJ, Moore PS. The epidemiology of HIV-associated Kaposi's sarcoma: the unravelling mystery. AIDS 1996;10(suppl A):S51-7.

11 Renwick N, Halaby T, Weverling GJ, et al. Seroconversion for human herpesvirus 8 during HIV infection is highty predictive of Kaposi's sarcoma. AIDS 1998;12:2481-8.

12 Di Alberti L, Piattelli A, Artese L, et al. Human herpesvirus 8 variants in sarcoid tissues. Lancet 1997;350:1655-61.

13 Koerner K, Cardoso M, Dengler T, et al. Estimated risk of transmission of hepatitis C virus by blood transfusion. Vox Sang 1998:74:213-16.

14 Schachter J. DFA, EIA, PCR, LCR and other technologies: what tests should be used for diagnosis of chlamydia infections? Immunol Invest 1997;26:157-61.

15 Rowley AH, Whitley RJ, Lakeman FD, et al. Rapid detection of herpes simplex virus DNA in cerebrospinal fluid of patients with herpes simplex encephalitis. Lancet 1990;335: patients

16 Aslanzadeh J, Osmon DR, Wilhelm MP, et al. A prospective study of the polymerase chain reaction for the detection of herpes simplex virus in cerebrospinal fluid submitted to the clinical virology laboratory. Mol Cell Probes 1992;6:367-73.

17 Guffond T, Dewilde A, Lobert P-E, et al. Significance and clinical relevance of the detection of herpes simplex virus DNA by the polymerase chain reaction in cerebrospinal fluid from patients with presumed encephalitis. Clin Infect Dis 1994;18:744-9.

18 Lakeman FD, Whitley RJ, and the National Institute of Allergy and Infectious Diseases Collaborative Antiviral Study Group. Diagnosis of herpes simplex encephalitis: application of polymerase chain reaction to cerebrospinal fluid from brain-biopsied patients and correlation with disease. F Infect Dis 1995;171:857-63.

19 Cinque P, Brytting M, Vago L, et al. Epstein-Barr virus DNA in cerebrospinal fluid from patients with AIDSrelated primary lymphoma of the central nervous system. related primary lymphoma

20 Tanel RE, Kao SY, Niemiec TM, et al. Prospective comparison of culture vs genome detection for diagnosis of enteroviral meningitis in childhood. Arch Pediatr Adolesc Me 1996;150:919-24.
21 Thoren A, Widell A. PCR for the diagnosis of enteroviral meningitis. Scand $\mathcal{F}$ Infect Dis 1994;26:249-54.

22 Yerly S, Gervaix A, Simonet V. Rapid and sensitive detection of enteroviruses in specimens from patients with aseptic meningitis. F Clin Microbiol 1996;34:199-201.

23 Mitchell PS, Espy MJ, Smith TF, et al. Laboratory diagnosis of central nervous system infections with herpes simplex virus by PCR performed with cerebrospinal fluid specimens. f Clin Microbiol 1997;35:2873-7.

24 Read SJ, Jeffery KJM, Bangham CRM. Aseptic meningitis and encephalitis: the role of PCR in the diagnostic laboratory. 7 Clin Microbiol 1997;35:691-6.

25 Echevarria JM, Casas I, Tenorio T, et al. Detection of varicella-zoster virus-specific DNA sequences in cerebrospinal fluid from patients with acute aseptic meningitis and no cutaneous lesions. F Med Virol 1994;43:331-5

26 Jeffery KJM, Read SJ, Peto TEA, et al. Diagnosis of viral infections of the central nervous system: clinical interpretation of PCR results. Lancet 1997:349:313-17.

27 Schlesinger Y, Tebas P, Gaudreault-Keener M, et al. Herpes simplex virus type 2 meningitis in the absence of genital esions: improved recognition with use of the polymerase chain reaction. Clin Infect Dis 1995;20:842-8.

28 Tedder DG, Ashley R, Tyler KL, et al. Herpes simplex virus infection as a cause of benign recurrent lymphocytic meningitis. Ann Intern Med 1994;121:334-8.

29 Read SJ, Kurtz JB. Laboratory diagnosis of common viral infections of the central nervous system by using a single multiplex PCR screening assay. f Clin Microbiol 1999;37: 1352-5.

30 The TH, van der Bij W, van den Berg AP, et al. Cytomegalovirus antigenaemia. Rev Infect Dis 1990;12(suppl 7): S734-44

31 Wolf DG, Spector SA. Early diagnosis of human cytomegalovirus disease in transplant recipients by DNA amplification in plasma. Transplantation 1993;56:330-4.

32 Burgart LJ, Heller MJ, Reznicek MJ, et al. Cytomegalovirus detection in bone marrow transplant patients with idiopathic pneumonitis. A clinicopathological study of the clinical utility of the polymerase chain reaction. Am f Clin Pathol 1991;96:572-6.

33 Evans PC, Soin A, Wreghitt TG, et al. Qualitative and semiquantitative polymerase chain reaction testing for cytomegalovirus DNA in serum allows prediction of CMV related disease in liver transplant recipients. 7 Clin Pathol 1998;51: 914-21.

34 Picciotto A, Campo N, Brizzolara R, et al. HCV-RNA levels play an important role independently of genotype in predicting response to interferon therapy. Eur $\mathcal{F}$ Gastroenterol Hepatol 1997;9:67-9.

35 Shiratori Y, Kato N, Yokosuka O, et al. Predictors of the efficacy of interferon therapy in chronic hepatitis $\mathrm{C}$ virus infection. Gastroenterology 1997;113:558-66.

36 Ho DD, Neumann AU, Perelson AS, et al. Rapid turnover of plasma virions and CD4 lymphocytes in HIV-1 infection. Nature 1995;373:123-6.

37 British HIV Association. Guidelines for antiretroviral treatment of HIV seropositive individuals. Lancet 1997;349: 1086-92.

38 Mellors JW, Rinaldo CR, Jr, Gupta P, et al. Prognosis in HIV-1 infection predicted by the quantity of virus in plasma. Science 1996;272:1167-70.

39 Bell H, Hellum K, Harthug S, et al. Genotype, viral load and age as independent predictors of treatment outcome of interferon-alpha $2 \mathrm{a}$ treatment in patients with chronic hepatitis C. Scand F Infect Dis 1997;29:17-22.

40 Mutimer D, Pillay D, Dragon E, et al. High pre-treatment serum hepatitis B virus titre predicts failure of lamivudine prophylaxis and grafts re-infection after liver transplantation. $\mathcal{f}$ Hepatol 1999;30:715-21.

41 Gerhold D, Rushmore T, Caskey CT. DNA chips: promising toys have become powerful tools. Trends Biochem Sci 1999;24:168-73.

42 Lipshutz RJ, Fodor SP, Gingeras TR, et al. High density synthetic oligonucleotide arrays. Nat Genet 1999;21(suppl $1): 20-4$.

43 Head SR, Parikh K, Rodgers YH, et al. Solid-phase sequence scanning for drug resistance detection in tuberculosis. Mol Cell Probes 1999;13:81-7. 